\title{
A Unique Case of Diffuse Histiocytic Proliferations Mimicking Metastatic Clear Cell Carcinoma in the Hydrocele Sac
}

\author{
Shirish S Chandanwale, Shruti S Vimal, Mohit Rajpal, Neha Mishra
}

Department of Pathology, Padmashree Dr. DY Patil Medical College, Pimpri, Pune, Maharashtra, India

Address for correspondence: Dr. Shirish S Chandanwale, E-mail: shirishchandanwale@gmail.com

\section{ABSTRACT}

Reactive histiocytic proliferations are extremely rare in paratesticular structures. Nodular histiocytic proliferations have been described in mesothelial-lined locations and only at few nonmesothelial sites. Diffuse histiocytic proliferations are described only in the pelvic peritoneum. We report the first case of diffuse histiocytic proliferation in the hydrocele sac of a 45-year-old man. Predominant histiocytes showed clear cytoplasm and signet ring-like change. Mucicarmin stain did not demonstrate mucin in the cytoplasm. Immunohistochemistry (IHC) staining showed nonspecific staining of these cells with carcinoembryonic antigen and negative staining with epithelial membrane antigen, pan-Cytokeratin, calretinin, cytokeratin 7, 20 and prostate-specific antigen. Strong diffuse cytoplasmic positivity for CD68 defined the mononuclear phagocyte nature of these cells. Diffuse histiocytic proliferations can occur in the hydrocele sac. Histochemical and IHC stainings are critical for accurate diagnosis and to avoid unnecessary surgery.

Key words: Clear cells, diffuse, histiocytes, Immunohistochemistry, signet ring cells

\section{INTRODUCTION}

seudo tumors and nonneoplastic cellular proliferations are rare in paratesticular structures. ${ }^{[1]}$ Histiocytic proliferations are uncommon reactive lesions and extremely rare in paratesticular structures. Usually, they form nodular collections. Nodular histiocytic proliferations (NHPs) have been described in mesothelial-lined locations ${ }^{[2-4]}$ and only at a few nonmesothelial sites. ${ }^{[2,5-7]}$ They are often confused with reactive, inflammatory and neoplastic lesions. Diffuse histiocytic proliferations are described only in the pelvic peritoneum of premenopausal women with endocervicosis. ${ }^{[8]}$ No literature had mention of these lesions in the hydrocele sac. We are documenting the first case of diffuse histiocytic proliferations in the hydrocele sac of an adult male showing predominantly clear to vacuolated cytoplasm and signet ring cell morphology.

\begin{tabular}{|l|l|}
\multicolumn{2}{|c|}{ Access this article online } \\
\hline Quick Response Code: & \\
\hline & Website: \\
\hline & \\
\hline
\end{tabular}

\section{CASE REPORT}

A 45-year-old man came with a complaint of scrotal swelling for 1 month and dull dragging pain for 7 days. He gave a history of minor trauma on the scrotum 15 days back. There was no other significant medical history. On local examination, the right hemiscrotum was tender $(7 \times 6 \mathrm{~cm})$ and did not transilluminate. The left hemiscrotum $(8.5 \times 7 \mathrm{~cm})$ was nontender and transilluminated. There was no erythema or ulcer of the scrotal skin. There was no inguinal lymphadenopathy. Provisional clinical diagnosis of the right hemiscrotal mass with left hydrocele was made. Ultrasonography of the scrotum diagnosed right scrotal pyocele and left scrotal hydrocele. Both the testis and epididymis were normal. Left-sided Jaboulay repair and right scrotal incision and drainage was performed. Preoperative and postoperative intravenous antibiotics were given.

Partially excised left hydrocele sac was received in multiple bits for histopathological examination [Figure 1a]. Histopathological examination showed fibrocollagenous wall of hydrocele sac that was diffusely infiltrated by large cells having clear to multivacuolated cytoplasm [Figure 1b]. Clear cells did not infiltrate outside the confines of the hydrocele sac wall. Nuclei 
were round to oval, showing moderate pleomorphism. Nuclei in few cells were pushed to the periphery with little distortion [Figure 1c]. Scant mitotic activity was seen. Few small foci of hemorrhages and lymphocytes were seen. There was no necrosis, significant inflammation, epitheloid granulomas or giant cells. Metastasis of clear cell carcinoma was suspected.

Cytoplasm of the cells did not stain with Mucicarmin stain [Figure 2a]. In immunohistochemistry (IHC) staining, cells were negative with epithelial membrane antigen (EMA), pan-Cytokeratin and calretinin [Figure 2b-d]. Cells showed strong diffuse cytoplasmic positivity with CD68 [Figure 3a]. Carcinoembryonic antigen (CEA) showed nonspecific staining [Figure $3 \mathrm{~b}$ ] and cytokeratin 7 (CK7), cytokeratin 20 (CK20) and prostate-specific antigen (PSA) were negative [Figure 3c-e]. Absence of significant inflammation, epitheloid granulomas and Michaelis-Gutman bodies in the histology sections ruled out xanthogranulomatous inflammation and malakoplakia. A computed tomography scan of the abdomen and thorax ruled out primary neoplastic lesions in the thorax and abdomen. Final histological diagnosis of diffuse histiocytic proliferation (reactive) in the hydrocele was made.

\section{DISCUSSION}

Reactive histiocytic proliferations are extremely rare in paratesticular tissues. These lesions are of a great interest because of their topography, and they might be relevant as a differential diagnosis. ${ }^{[1]}$ Previously, these lesions were referred to as nodular mesothelial hyperplasia. Now, IHC has established the cell of origin as histiocytic rather than mesothelial. ${ }^{[2]}$ Usually, they form nodular proliferations and are referred to as NHPs). ${ }^{[7]}$ NHP has been reported in mesothelial-lined locations such as the pleura, pericardium, peritoneum and hernia sac. ${ }^{[2-4]}$ Nonmesothelial sites like the urinary bladder, endometrium and lungs are very rarely involved. ${ }^{[2,5-7]}$ These lesions can be confused with a variety of reactive, inflammatory or neoplastic conditions. They are mostly made up of nodular aggregates of histiocytes. Histiocytes have moderate amount of eosinophilic cytoplasm, ovoid to slightly lobulated vesicular nuclei and inconspicuous nucleoli. These lesions may contain variable proportions of histiocytes with vacuolated to clear cytoplasm, and could be misinterpreted as metastatic carcinoma. ${ }^{[4,5,9]}$

Ruffolo reported diffuse histiocytic proliferations mimicking mesothelial hyperplasia in the pelvic peritoneum of premenopausal women. ${ }^{[8]}$ Fukunaga and Iwaki observed clear cell and signet ring cell changes in NHP of the

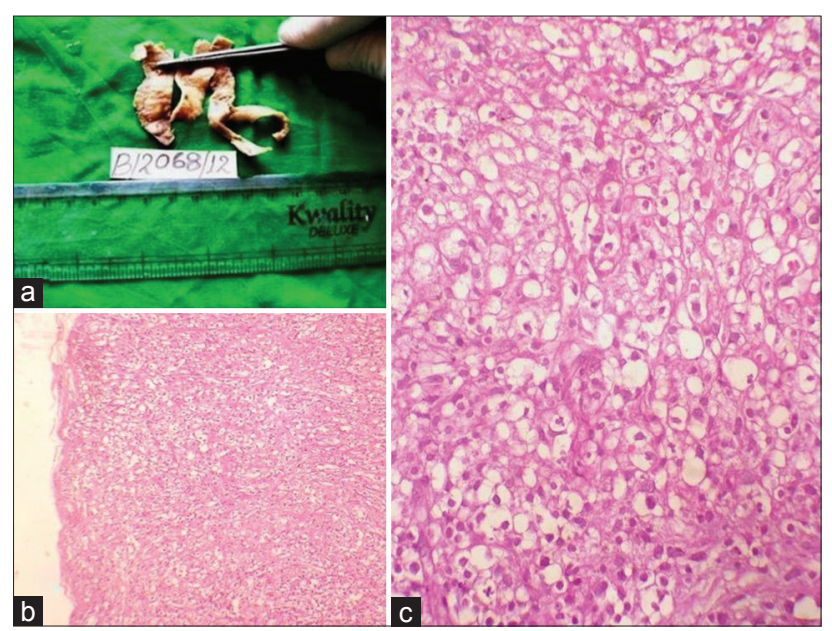

Figure 1: (a) Hydrocele sac wall. (b) Hydrocele sac wall diffusely infiltrated by histiocytes having clear to multivacuolated cytoplasm $(\mathrm{H}$ and $\mathrm{E}$, $\times 100$ ). (c) Large cells with moderate nuclear pleomorphism and clear/ vacuolated cytoplasm $(\mathrm{H}$ and $\mathrm{E}, \times 400)$

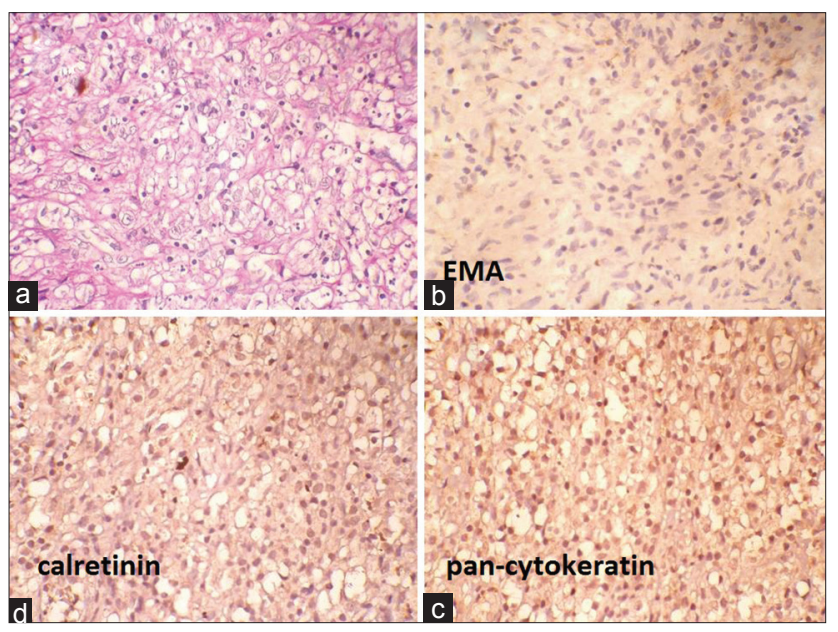

Figure 2: (a) Mucicarmin-negative cytoplasm of the histiocytes (Mucicarmin, $\times 400$ ). (b-d) Negative epithelial membrane antigen, pan-Cytokeratin and calretinin (immunohostochemistry, $\times 400$ )

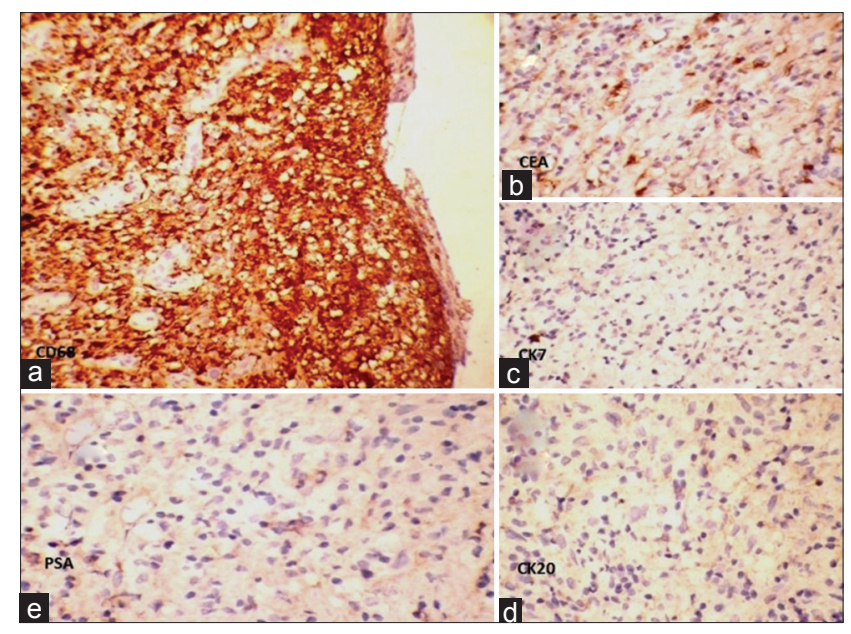

Figure 3: Histiocytes show (a) strong diffuse cytoplasmic positivity with CD 68 (IHC, $\times 100)$. (b) Nonspecific staining with carcinoembryonic antigen (IHC, $\times 400$ ). (c-e) Negative staining with cytokeratin 7 , cytokeratin 20 and prostate-specific antigen $(\mathrm{IHC}, \times 400)$

Journal of Laboratory Physicians / Jan-Jun 2014 / Vol-6 / Issue-1 
endometrium..$^{[9]}$ In our case, the hydrocele sac wall was diffusely infiltrated by cells having predominantly clear to vacuolated cytoplasm, and few cells were showing signet ring cell-like change. Occasionally, reactive histiocytes are known to show moderate nuclear pleomorphism and high mitotic activity. ${ }^{[5,10]}$

Metastasis of clear cell carcinoma was thought at the first instance. Mucicarmin staining was negative. In IHC staining, cells were negative for pan-Cytokeratin and EMA, which ruled out metastasis of epithelial malignancy. The hydrocele sac is a mesothelial-lined surface. Negative staining with calretinin ruled out mesothelial nature of the cells. Negative CEA, CK7 and CK20 staining of the cells ruled out metastasis of the gastrointestinal adenocarcinoma and majority of the other ductal carcinomas. PSA-negative staining ruled out metastasis of prostate carcinoma. Strong diffuse cytoplasmic positivity with CD68 revealed the mononuclear phagocyte nature of the cells (macrophages). Although signet ring cells were once considered to be synonymous with adenocarcinoma, they are now known to occur in a variety of noncarcinomatous lesions and in nonneoplastic lesions. ${ }^{[1]}$

Development of reactive histiocytic hyperplasia probably results from the irritation of mesothelial lining due to infection or surgery (trauma), which leads to aggregation of histiocytes. Accurate diagnosis of such lesion is essential to prevent erroneous diagnosis and unnecessary treatment.

\section{CONCLUSION}

Reactive histiocytic proliferations are extremely rare. NHPs have been described at mesothelial and few nonmesothelial sites. Diffuse histiocytic proliferations are described only in the pelvic peritoneum. They can occur in the hydrocele sac. Predominant histiocytes can have clear to vacuolated cytoplasm and signet ring-like morphology. Histochemical and IHC staining are critical for accurate diagnosis and to avoid unnecessary surgery.

\section{REFERENCES}

1. Algaba F, Mikuz G, Boccon-Gibod L, Trias I, Arce Y, Montironi R, et al. Pseudoneoplastic lesions of the testis and paratesticular structures. Virchows Arch 2007;451:987-97.

2. Ordóñez HG, Ro JY, Ayala AG. Lesions described as nodular mesothelial hyperplasia are primarily composed of histiocytes. Am J Surg Pathol 1998;22:285-92.

3. Rosai J, Dehner LP. Nodular mesothelial hyperplasia in hernia sacs: A benign reactive condition simulating a neoplastic process. Cancer 1975;35:165-75.

4. Rao S, Khurana N, Mohta A. Nodular histiocytic proliferation in hernial sac: A potential diagnostic pitfall. Indian J Pathol Microbiol 2012;55:126.

5. Chikkamuniyappa S, Herrick J, Jagirdar JS. Nodular histiocytic/mesothelial hyperplasia: A potential pitfall. Ann Diagn Pathol 2004;8:115-20.

6. Wang CW, Colby TV. Histiocytic lesions and proliferations in the lung. Semin Diagn Pathol2007;24:162-82.

7. Chan JK, Loo KT, Yau BK, Lam SY. Nodular histiocytic/mesothelial hyperplasia: A lesion potentially mistaken for a neoplasm in transbronchial biopsy. Am J Surg Pathol1997;21:658-63.

8. Ruffolo R, Suster S. Diffuse histiocytic proliferation mimicking mesothelial hyperplasia in endocervicosis of the female pelvic peritoneum. Int J Surg Pathol1993;1:101-5.

9. Fukunaga M, Iwaki S. Nodular histiocytic hyperplasia of the endometrium. Arch Pathol Lab Med2004;128:1032-4.

10. Kim KR, Lee YH, Ro JY. Nodular histiocytic hyperplasia of the endometrium. Int J Gynecol Pathol2002;21:141-6.

11. Iezzoni JC, Mills SE. Nonneoplastic endometrial signet-ring cells. Vacuolated decidual cells and stromal histiocytes mimicking adenocarcinoma. Am J Clin Pathol 2001;115:249-55.

How to cite this article: Chandanwale SS, Vimal SS, Rajpal M, Mishra $\mathrm{N}$. A Unique case of diffuse histiocytic proliferations mimicking metastatic clear cell carcinoma in the hydrocele sac. J Lab Physicians 2014;6:43-5.

Source of Support: Nill. Conflict of Interest: None declared. 